\title{
The probability of planarity of a random graph near the critical point
}

\author{
Marc Noy 1 \\ Vlady Ravelomanana ${ }^{2}$ \\ Juanjo Rués|⿰亻三 \\ ${ }^{1}$ Dep. de Matemàtica Aplicada II. Universitat Politècnica de Catalunya. Jordi Girona 1-3, Barcelona. Spain \\ ${ }^{2}$ Liafa UMR CNRS 7089, Université Denis Diderot, Paris, France \\ ${ }^{3}$ Instituto de Ciencias Matemáticas. Nicolás Cabrera 13-15, Madrid. Spain
}

\begin{abstract}
Erdôs and Rényi conjectured in 1960 that the limiting probability $p$ that a random graph with $n$ vertices and $M=n / 2$ edges is planar exists. It has been shown that indeed $p$ exists and is a constant strictly between 0 and 1. In this paper we answer completely this long standing question by finding an exact expression for this probability, whose approximate value turns out to be $p \approx 0.99780$.
\end{abstract}

More generally, we compute the probability of planarity at the critical window of width $n^{2 / 3}$ around the critical point $M=n / 2$. We extend these results to some classes of graphs closed under taking minors. As an example, we show that the probability of being series-parallel converges to 0.98003 .

Our proofs rely on exploiting the structure of random graphs in the critical window, obtained previously by Janson, Łuczak and Wierman, by means of generating functions and analytic methods. This is a striking example of how analytic combinatorics can be applied to classical problems on random graphs.

Résumé. Erdős et Rényi ont conjecturé en 1960 que la probabilité limite $p$ qu'un graphe aléatoire avec $n$ sommets et $M=n / 2$ arêtes soit planaire existe. Il a été prouvé qu'en fait $p$ existe et est une constante comprise strictement entre 0 et 1 . Dans ce travail nous fermons complètement cette question en trouvant l'expression exacte pour cette probabilité, dont la valeur approchée s'avère être $p \approx 0.99780$.

Plus genéralement, nous calculons la probabilité qu'un graphe soit planaire dans la fenêtre critique de largeur $n^{2 / 3}$ autour du point critique $M=n / 2$. Nous étendons ces resultats à différentes classes de graphes closes par exclusion de mineurs. A titre d'exemple, nous montrons que la probabilité d'être série-parallèle converge vers 0.98003.

Nos preuves exploitent la structure des graphes aléatoires dans la fenêtre critique, décrite précedemment par Janson, Łuczak et Wierman, en utilisant les séries génératrices et des méthodes analytiques. Cet exemple notable montre que la combinatoire analytique peut être utilisée pour des problèmes classiques de graphes aléatoires.

Keywords: random graphs, planar cubic multigraphs, analytic combinatorics

\footnotetext{
${ }^{\dagger}$ Partially supported by grants MTM2011-24097 and DGR2009-SGR1040.

$\ddagger$ Partially supported by grants JAE-DOC (CSIC), MTM2011-22851 and SEV-2011-0087.
} 


\section{Introduction}

The random graph model $G(n, M)$ assigns uniform probability to graphs on $n$ labelled vertices with $M$ edges. A fundamental result of Erdős and Rényi (1960) is that the random graph $G(n, M)$ undergoes an abrupt change when $M$ is around $n / 2$, the value for which the average vertex degree is equal to one. When $M=c n / 2$ and $c<1$, almost surely the connected components are all of order $O(\log n)$, and are either trees or unicyclic graphs. When $M=c n / 2$ and $c>1$, almost surely there is a unique giant component of size $\Theta(n)$. We direct to reader to the reference texts of Bollobás (1985) and Janson et al. (2000) for a detailed discussion of these facts.

We concentrate on the so-called critical window namely $M=\frac{n}{2}\left(1+\lambda n^{-1 / 3}\right)$, where $\lambda$ is a real number, identified by Bollobás $(1984 \mathrm{a} b)$. Let us recall that the excess of a connected graph is the number of edges minus the number of vertices. A connected graph is complex if it has positive excess. As $\lambda \rightarrow-\infty$, complex components disappear and only trees and unicyclic components survive, and as $\lambda \rightarrow+\infty$, components with unbounded excess appear. A thorough analysis of the random graph in the critical window can be found in Janson et al. (1993) and Łuczak et al. (1994), which constitute our basic references.

For each fixed $\lambda$, we denote the random graph $G\left(n, \frac{n}{2}\left(1+\lambda n^{-1 / 3}\right)\right)$ by $G(\lambda)$. The core $C(\lambda)$ of $G(\lambda)$ is obtained by repeatedly removing all vertices of degree one from $G(\lambda)$. The kernel $K(\lambda)$ is obtained from $C(\lambda)$ by replacing all maximal paths of vertices of degree two by single edges. The parameter $n$ is implicitly assumed in all the previous definitions. The graph $G(\lambda)$ satisfies asymptotically almost surely several fundamental properties, that were established by Łuczak et al. (1994) by a subtle simultaneous analysis of the $G(n, M)$ and the $G(n, p)$ models.

1. The number of complex components is bounded.

2. Each complex component has size of order $n^{2 / 3}$, and the largest suspended tree in each complex component has size of order $n^{2 / 3}$.

3. $C(\lambda)$ has size of order $n^{1 / 3}$ and maximum degree three, and the distance between two vertices of degree three in $C(\lambda)$ is of order $n^{1 / 3}$.

4. $K(\lambda)$ is a cubic (3-regular) multigraph of bounded size.

The key property for us is the last one. It implies that asymptotically almost surely the components of $G(\lambda)$ are trees, unicyclic graphs, and those obtained from a cubic multigraph $K$ by attaching rooted trees to the vertices of $K$, and attaching ordered sequences of rooted trees to the edges of $K$. Some care is needed here, since the resulting graph may not be simple, but asymptotically this can be accounted for.

It is clear that $G(\lambda)$ is planar if and only if the kernel $K(\lambda)$ is planar. Then by counting planar cubic multigraphs it is possible to estimate the probability that $G(\lambda)$ is planar. To this end we use generating functions. The trees attached to $K(\lambda)$ are encoded by the generating function $T(z)$ of rooted trees, and complex analytic methods are used to estimate the coefficients of the corresponding series. This allows us to determine the exact probability

$$
p(\lambda)=\lim _{n \rightarrow \infty} \operatorname{Pr}\left\{G\left(n, \frac{n}{2}\left(1+\lambda n^{-1 / 3}\right)\right) \text { is planar }\right\} .
$$

In particular, we obtain $p(0) \approx 0.99780$.

This approach was initiated in the seminal paper of Flajolet et al.(1989), where the authors determined the threshold for the appearance of the first cycles in $G(n, M)$. A basic feature in Flajolet et al. (1989) 
is to estimate coefficients of large powers of generating functions using Cauchy integrals and the saddle point method. This path was followed by Janson et al. (1993), obtaining a wealth of results on $G(\lambda)$. Of particular importance for us is the determination in Janson et al. (1993) of the limiting probability that $G(\lambda)$ has given excess. The approach in Łuczak et al. (1994) is more probabilistic and has as starting point the classical estimates by Wright (1980) on the number of connected graphs with fixed excess. The range of these estimates was extended by Bollobás (1984a) and more recently the analysis was refined by Flajolet et al. (2004), by giving complete asymptotic expansions in terms of the Airy function. Very recently, the question of planarity has been analyzed in a wider setting of random subgraphs of a given graph by Frieze and Krivelevich.

The paper is organized as follows. In Section 2 we present the basic lemmas needed in the sequel. In Section 3 we compute the number of cubic planar multigraphs, suitably weighted, where we follow Kang and Łuczak (2012). In Section 4 we compute the exact probability that the random graph $G(\lambda)$ is planar as a function of $\lambda$. We generalize this result by determining the probability that $G(\lambda)$ belongs to a minorclosed class of graphs in several cases of interest.

We close this introduction with a remark. The problem of 2-satisfiability presents a striking analogy with the random graph process. Given $n$ Boolean variables and a conjunctive formula of $M$ clauses, each involving two literals, the problem is to determine the probability that the formula is satisfiable when $M$ grows with $n$. The threshold has been established at $M=n$ and the critical window is also of width $n^{2 / 3}$; see Bollobás et al. (2001). However the exact probability of satisfiability when the number of clauses is $n\left(1+\lambda n^{-1 / 3}\right)$ has not been determined, and appears to be a more difficult problem.

\section{Preliminaries}

All graphs in this paper are labelled. The size of a graph is its number of vertices. A multigraph is a graph with loops and multiple edges allowed.

We recall that the exponential generating function $T(z)$ of rooted trees satisfies

$$
T(z)=z e^{T(z)} .
$$

Using Lagrange's inversion (see Flajolet and Sedgewick (2009)), one recovers the classical formula $n^{n-1}$ for the number of rooted labelled trees. The generating function for unrooted trees is

$$
U(z)=T(z)-\frac{T(z)^{2}}{2} .
$$

This can be proved by integrating the relation $T(z)=z U^{\prime}(z)$, or more combinatorially using the dissimilarity theorem for trees (see Otter (1948)).

A graph is unicyclic if it is connected and has a unique cycle. A unicyclic graph can be seen as an undirected cycle of length at least three to which we attach a sequence of rooted trees. Since the directed cycle construction corresponds algebraically to $\log (1 /(1-z))$ (see Flajolet and Sedgewick (2009)), the generating function is

$$
V(z)=\frac{1}{2}\left(\log \frac{1}{1-T(z)}-T(z)-\frac{T(z)^{2}}{2}\right) .
$$

Graphs whose components are unicyclic are given by the exponential formula:

$$
e^{V(z)}=\frac{e^{-T(z) / 2-T(z)^{2} / 4}}{\sqrt{1-T(z)}} .
$$


The following result, which is fundamental for us, is proved in Theorem 4 of tuczak et al. (1994) by a careful analysis of the structure of complex components in $G(\lambda)$. We say that a property $\mathcal{P}$ holds asymptotically almost surely (a.a.s.) in $G(n, M)$ if the probability that $\mathcal{P}$ is satisfied tends to one as $n \rightarrow \infty$. Recall that $G(\lambda)=G\left(n, \frac{n}{2}\left(1+\lambda n^{-1 / 3}\right)\right)$.

Lemma 1 For each $\lambda$, the kernel of $G(\lambda)$ is a.a.s. a cubic multigraph.

Given a cubic multigraph $M$ with $a$ loops, $b$ double edges and $c$ triple edges, define its weight as

$$
w(M)=2^{-a} 2^{-b} 6^{-c} .
$$

This weight (called the compensation factor in Janson et al.(1993)), has the following explanation. When we substitute edges of the kernel by sequences of rooted trees, a loop has two possible orientations that give rise to the same graph. A double (triple) edge can be permuted in two (six) ways, again producing the same graph. From now on, all multigraphs we consider are weighted, so that we omit the qualifier. The following lemma is proved in Janson et al. (1993) using a combination of guessing and recurrence relations. The proof we give appears in Chapter 2 of the book Bollobás (1985).

Lemma 2 The number $E_{r}$ of cubic multigraphs with $2 r$ vertices is equal to

$$
E_{r}=\frac{(6 r) !}{(3 r) ! 2^{3 r} 6^{2 r}}
$$

Proof: A cubic multigraph can be modeled as a pairing of darts (half-edges), 3 for each vertex, with a total of $6 r$ darts. The number of such pairings is $(6 r) ! /\left((3 r) ! 2^{3 r}\right)$. However, we have to divide by the number $6^{2 r}$ of ways of permuting each of the $2 r$ triples of darts. The weight takes care exactly of the number of times a cubic multigraph is produced in this process.

The next result is essentially proved in Janson et al. (1993). Here we present a concise proof. We denote by $\left[z^{n}\right] A(z)$ the coefficient of $z^{n}$ in the power series $A(z)$.

Lemma 3 The number $g(n, M, r)$ of simple graphs with $n$ vertices, $M$ edges and cubic kernel of size $2 r$ satisfies

$$
g(n, M, r) \leq n !\left[z^{n}\right] \frac{U(z)^{n-M+r}}{(n-M+r) !} e^{V(z)} \frac{E_{r}}{(2 r) !} \frac{T(z)^{2 r}}{(1-T(z))^{3 r}}
$$

and

$$
g(n, M, r) \geq n !\left[z^{n}\right] \frac{U(z)^{n-M+r}}{(n-M+r) !} e^{V(z)} \frac{E_{r}}{(2 r) !} \frac{T(z)^{8 r}}{(1-T(z))^{3 r}} .
$$

Proof: Such a graph is the union of a set of $s$ unrooted trees, a set of unicyclic graphs, and a cubic multigraph $K$ with a rooted tree attached to each vertex of $K$ and a sequence (possibly empty) of rooted trees attached to each edge of $K$. Let us see first that $s=n-M+r$. Indeed, the final excess of edges over vertices must be $M-n$. Each tree component contributes with excess -1 , each unicyclic component with excess 0 , and $K$ (together with the attached trees) with excess $r$. Hence $M-n=-s+r$.

The first two factors $U(z)^{n-M+r} /(n-M+r)$ ! and $e^{V(z)}$ on the right-hand side of the inequalities encode the set of trees and unicyclic components. The last part encodes the kernel $K$. It has $2 r$ vertices and 
is labelled, hence the factor $E_{r} /(2 r)$ !; the weighting guarantees that each graph contributing to $g(n, M, r)$ is counted exactly once. The trees attached to the $2 r$ vertices give a factor $T(z)^{2 r}$. The sequences of trees attached to the $3 r$ edges give each a factor $1 /(1-T(z))$. However, this allows for the empty sequence and the resulting graph may not be simple, so we get only an upper bound. To guarantee that the final graph is simple we take sequences of length at least two, encoded by $T(z)^{2} /(1-T(z))$ (length one is enough for multiple edges of $K$, but length two is needed for loops). Since this misses some graphs, we get a lower bound.

The following technical result is essentially Lemma 3 from Janson et al. (1993).

Lemma 4 Let $M=\frac{n}{2}\left(1+\lambda n^{-1 / 3}\right)$. Then for any fixed a and integer $r>0$ we have

$$
\frac{n !}{\left(\begin{array}{c}
n \\
2 \\
M
\end{array}\right)}\left[z^{n}\right] \frac{U(z)^{n-M+r}}{(n-M+r) !} \frac{T(z)^{a}}{(1-T(z))^{3 r}} e^{V(z)}=\sqrt{2 \pi} A\left(3 r+\frac{1}{2}, \lambda\right)\left(1+O\left(\frac{1+\lambda^{4}}{n^{1 / 3}}\right)\right)
$$

uniformly for $|\lambda| \leq n^{1 / 12}$, where

$$
A(y, \lambda)=\frac{e^{-\lambda^{3} / 6}}{3^{(y+1) / 3}} \sum_{k \geq 0} \frac{\left(\frac{1}{2} 3^{2 / 3} \lambda\right)^{k}}{k ! \Gamma((y+1-2 k) / 3)} .
$$

We omit the proof, which is based on relating the left-hand side of Equation (1) to the integral representation of $A(y, \lambda)$ defined in Equation (10.7) of Janson et al. (1993):

$$
A(y, \lambda)=\frac{1}{2 \pi i} \int_{\Pi} s^{1-y} e^{K(\lambda, s)} d s
$$

where $K(\lambda, s)$ is the polynomial

$$
K(\lambda, s)=\frac{(s+\lambda)^{2}(2 s-\lambda)}{6}=\frac{s^{3}}{3}+\frac{\lambda s^{2}}{2}-\frac{\lambda^{3}}{6}
$$

and $\Pi$ is a suitable path in the complex plane.

It is important to notice that in the previous lemma the final asymptotic estimate does not depend on the choice of $a$. The next result is a direct consequence and can be found as Formula (13.17) in Janson et al. (1993).

Lemma 5 The limiting probability that the random graph $G(\lambda)$ has a cubic kernel of size $2 r$ is equal to

$$
\sqrt{2 \pi} e_{r} A\left(3 r+\frac{1}{2}, \lambda\right)
$$

where $e_{r}=E_{r} /(2 r)$ ! ( $E_{r}$ is defined in Lemma 2) and $A(y, \lambda)$ is as in the previous lemma.

In particular, for $\lambda=0$ the limiting probability is

$$
\sqrt{\frac{2}{3}}\left(\frac{4}{3}\right)^{r} e_{r} \frac{r !}{(2 r) !}
$$


Proof: Using the notation of Lemma 3 , the probability for a given $n$ is by definition

$$
\frac{g(n, M, r)}{\left(\begin{array}{c}
n \\
2 \\
M
\end{array}\right)}
$$

Lemma 3 gives upper and lower bounds for this probability, and using Lemma 4 we see that both bounds agree in the limit and are equal to

$$
\frac{E_{r}}{(2 r) !} \sqrt{2 \pi} A\left(3 r+\frac{1}{2}, \lambda\right)
$$

thus proving the result. A key point is that the discrepancy between the factors $T(z)^{2 r}$ and $T(z)^{8 r}$ in the bounds for $g(n, M, r)$ does not affect the limiting value of the probability.

Notice that if we replace the $e_{r}$ by the numbers $g_{r}$ arising by counting planar cubic multigraphs, we obtain immediately the probability that $G(\lambda)$ has a cubic planar kernel of size $2 r$. Since $G(\lambda)$ is planar if and only if its kernel is planar, we can use this fact to compute the probability of $G(\lambda)$ being planar. But first we must compute $g_{r}$.

\section{Planar cubic multigraphs}

In this section we compute the numbers $G_{r}$ of cubic weighted planar multigraphs of size $2 r$. All multigraphs are labelled in vertices, hence the counting formulas are exponential in this parameter. The associated generating function has been obtained recently in Kang and Łuczak (2012) (generalizing the enumeration of simple cubic graphs in Bodirsky et al. (2007)), but their derivation contains some minor errors. They do not affect the correctness of Kang and Łuczak (2012), since the asymptotic estimates needed by the authors are still valid. However, for the computations that follow we need the exact values. The next result is from Kang and Łuczak (2012), the corrections are detailed below.

Lemma 6 Let $G_{1}(z)$ be the generating function of connected cubic planar multigraphs. Then $G_{1}(z)$ is determined by the following system of equations:

$$
\begin{array}{ll}
3 z \frac{d G_{1}(z)}{d z} & =D(z)+C(z) \\
B(z) & =\frac{z^{2}}{2}(D(z)+C(z))+\frac{z^{2}}{2} \\
C(z) & =S(z)+P(z)+H(z)+B(z) \\
D(z) & =\frac{B(z)^{2}}{z^{2}} \\
S(z) & =C(z)^{2}-C(z) S(z) \\
P(z) & =z^{2} C(z)+\frac{1}{2} z^{2} C(z)^{2}+\frac{z^{2}}{2} \\
2(1+C(z)) H(z) & =u(z)(1-2 u(z))-u(z)(1-u(z))^{3} \\
z^{2}(C(z)+1)^{3} & =u(z)(1-u(z))^{3} .
\end{array}
$$


The generating functions $B(z), C(z), D(z), S(z), P(z)$ and $H(z)$ correspond to distinct families of edge-rooted cubic planar graphs, and $u(z)$ is an algebraic function related to the enumeration of 3connected cubic planar graphs (dually, 3-connected triangulations).

The corrections with respect to Kang and Łuczak (2012) are the following. In the first equation a term $-7 z^{2} / 24$ has been removed. In the second and sixth equations we have replaced a term $z^{2} / 4$ by $z^{2} / 2$. In the fourth equation we have removed a term $-z^{2} / 16$. For the combinatorial interpretation of the various generating functions and the proof of the former equations we refer to Kang and Łuczak (2012). Notice that eliminating $u(z)$ from the last two equations we obtain a relation between $C(z)$ and $H(z)$. This relation can be used to obtain a single equation satisfied by $C(z)$, by eliminating $S(z), P(z), H(z)$, $D(z)$ and $B(z)$ from the first equations. We reproduce it here in case the reader wishes to check our computations.

$$
\begin{aligned}
& 1048576 z^{6}+1034496 z^{4}-55296 z^{2}+ \\
& \left(9437184 z^{6}+6731264 z^{4}-1677312 z^{2}+55296\right) C+ \\
& \left(37748736 z^{6}+18925312 z^{4}-7913472 z^{2}+470016\right) C^{2}+ \\
& \left(88080384 z^{6}+30127104 z^{4}-16687104 z^{2}+1622016\right) C^{3}+ \\
& \left(132120576 z^{6}+29935360 z^{4}-19138560 z^{2}+2928640\right) C^{4}+ \\
& \left(132120576 z^{6}+19314176 z^{4}-12429312 z^{2}+2981888\right) C^{5}+ \\
& \left(88080384 z^{6}+8112384 z^{4}-4300800 z^{2}+1720320\right) C^{6}+ \\
& \left(37748736 z^{6}+2097152 z^{4}-614400 z^{2}+524288\right) C^{7}+ \\
& \left(9437184 z^{6}+262144 z^{4}+65536\right) C^{8}+1048576 C^{9} z^{6}=0 .
\end{aligned}
$$

The first terms are

$$
C(z)=z^{2}+\frac{25}{8} z^{4}+\frac{59}{4} z^{6}+\frac{11339}{128} z^{8}+\cdots
$$

This allows us to compute $B(z), D(z), S(z), P(z)$ and $H(z)$, hence also $G_{1}(z)$. The first coefficients of $G_{1}(z)$ are as follows.

$$
G_{1}(z)=\frac{5}{24} z^{2}+\frac{5}{16} z^{4}+\frac{121}{128} z^{6}+\frac{1591}{384} z^{8}+\cdots
$$

Using the set construction, the generating function $G(z)$ for cubic planar multigraphs is then

$$
G(z)=e^{G_{1}(z)}=\sum_{r=0}^{\infty} G_{r} \frac{z^{2 r}}{(2 r) !}=1+\frac{5}{24} z^{2}+\frac{385}{1152} z^{4}+\frac{83933}{82944} z^{6}+\frac{35002561}{7962624} z^{8}+\cdots
$$

where $G_{r}$ is the number of planar cubic multigraphs with $2 r$ vertices. This coincides with the generating function for all cubic (non-necessarily planar) multigraphs up to the coefficient of $z^{4}$. The first discrepancy is in the coefficient of $z^{6}$. The difference between the coefficients is $1 / 72=10 / 6$ !, corresponding to the 10 possible ways of labelling $K_{3,3}$, the unique non-planar cubic multigraph on six vertices.

\section{Probability of planarity and generalizations}

Let $G$ be a graph with a cubic kernel $K$. Then clearly $G$ is planar if and only if $K$ is planar, and we can compute the probability that $G(n, M)$ is planar by counting over all possible planar kernels. 
Theorem 7 Let $g_{r}(2 r)$ ! be the number of cubic planar multigraphs with $2 r$ vertices. Then the limiting probability that the random graph $G\left(n, M=\frac{n}{2}\left(1+\lambda n^{-1 / 3}\right)\right)$ is planar is

$$
p(\lambda)=\sum_{r \geq 0} \sqrt{2 \pi} g_{r} A\left(3 r+\frac{1}{2}, \lambda\right) .
$$

In particular, the limiting probability that $G\left(n, \frac{n}{2}\right)$ is planar is

$$
p(0)=\sum_{r \geq 0} \sqrt{\frac{2}{3}}\left(\frac{4}{3}\right)^{r} g_{r} \frac{r !}{(2 r) !} \approx 0.99780 .
$$

Proof: The same analysis as in Section 2 shows that $\sqrt{2 \pi} g_{r} A\left(3 r+\frac{1}{2}, \lambda\right)$ is the probability that the kernel is planar and has $2 r$ vertices. Summing over all possible $r$, we get the desired result.

As already mentioned, in Erdôs and Rényi (1960) it was conjectured that $p(0)$ exists and $0<p(0)<1$. This was proved in Łuczak et al. (1994), showing that $p(\lambda)$ exists for all $\lambda$ and that $0<p(\lambda)<1$. The bounds in Janson et al. (1993) for $p(0)$ are

$$
0.98707<p(0)<0.99977
$$

obtained by considering connected cubic multigraphs with at most six vertices. We remark that in Stepanov (1988) is shown that $p(\lambda)<1$ for $\lambda \leq 0$ (without actually establishing the existence of the limiting probability). The function $p(\lambda)$ is plotted in Figure 1 . As expected, $p(\lambda)$ is close to 1 when $\lambda \rightarrow-\infty$ and close to 0 when $\lambda \rightarrow \infty$. For instance, $p(-3) \approx 1-1.02 \cdot 10^{-7}$ and $p(5) \approx 4.9 \cdot 10^{-7}$.

Besides planar graphs, one can consider other classes of graphs. Let $\mathcal{G}$ be a class of graphs closed under taking minors, that is, if $H$ is a minor of $G$ and $G \in \mathcal{G}$, then $H \in \mathcal{G}$. If $H_{1}, \cdots, H_{k}$ are the excluded minors of $\mathcal{G}$, then we write $\mathcal{G}=\operatorname{Ex}\left(H_{1}, \ldots, H_{k}\right)$. (By the celebrated theorem of Robertson and Seymour, the number of excluded minors is finite, but we do not need this deep result here). The following result generalizes the previous theorem.

Theorem 8 Let $\mathcal{G}=E x\left(H_{1}, \ldots, H_{k}\right)$ and assume all the $H_{i}$ are 3-connected. Let $h_{r}(2 r) !$ be the number of cubic multigraphs in $\mathcal{G}$ with $2 r$ vertices. Then the limiting probability that the random graph $G(n, M=$ $\left.\frac{n}{2}\left(1+\lambda n^{-1 / 3}\right)\right)$ is in $\mathcal{G}$ is

$$
p_{\mathcal{G}}(\lambda)=\sum_{r \geq 0} \sqrt{2 \pi} h_{r} A\left(3 r+\frac{1}{2}, \lambda\right) .
$$

In particular, the limiting probability that $G\left(n, \frac{n}{2}\right)$ is in $\mathcal{G}$ is

$$
p_{\mathcal{G}}(0)=\sum_{r \geq 0} \sqrt{\frac{2}{3}}\left(\frac{4}{3}\right)^{r} h_{r} \frac{r !}{(2 r) !}
$$

Moreover, for each $\lambda$ we have

$$
0<p_{\mathcal{G}}(\lambda)<1
$$


Proof: If all the $H_{i}$ are 3-connected, then clearly a graph is in $\mathcal{G}$ if and only its kernel is in $\mathcal{G}$. The probability $p_{\mathcal{G}}(\lambda)$ is then computed as in Theorem 7 . It is positive since $\mathcal{G}$ contains all trees and unicyclic graphs, which contribute with positive probability (although tending to 0 as $\lambda \rightarrow \infty$ ). To prove that it is less than one, let $t$ be the smallest size of the excluded minors $H_{i}$. By splitting vertices it is easy to construct cubic graphs containing $K_{t+1}$ as a minor, hence $G(\lambda)$ contains $K_{t+1}$ as a minor with positive probability (alternatively, see the argument at the end of Łuczak et al. (1994)). It follows that $1-p_{\mathcal{G}}(\lambda)>$ 0 .

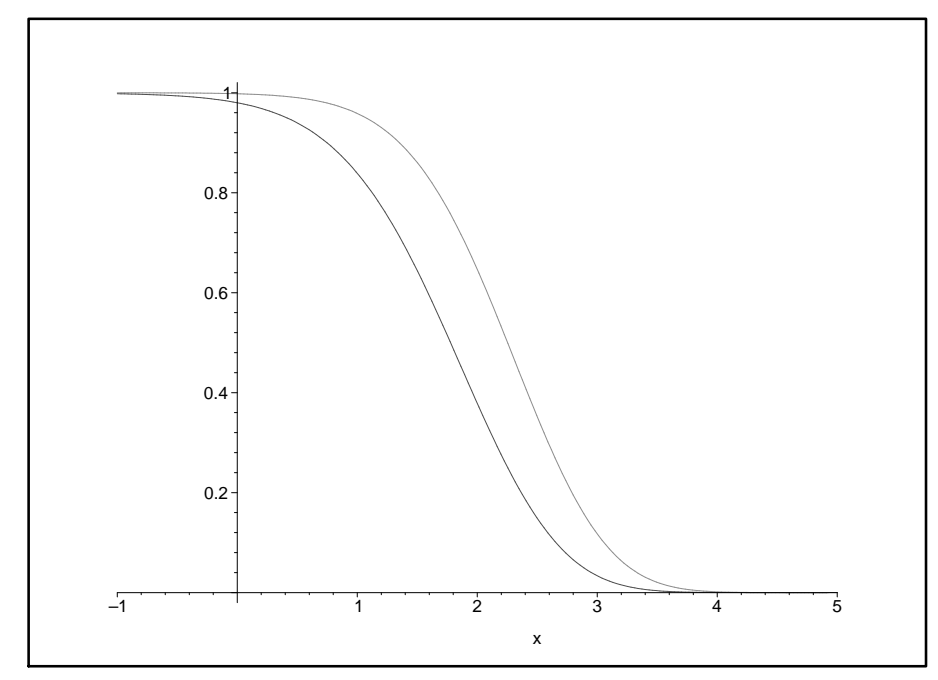

Fig. 1: The probability of $G(\lambda)$ being planar and of being series-parallel are both plotted for $\lambda \in[-1,4]$. The function on top corresponds to the planar case.

In some cases of interest we are able to compute the numbers $h_{r}$ explicitly. Let $\mathcal{G}=\operatorname{Ex}\left(K_{4}\right)$ be the class of series-parallel graphs. The same system of equations as in Lemma 6 holds for series-parallel graphs with the difference that now $H(z)=0$ (this is due to the fact that there are no 3-connected series-parallel graphs). The generating function for cubic series-parallel multigraphs can be computed as

$$
G_{\mathrm{sp}}(z)=1+\frac{5}{24} z^{2}+\frac{337}{1152} z^{4}+\frac{55565}{82944} z^{6}+\frac{15517345}{7962624} z^{8}+\cdots
$$

For instance, $\left[z^{4}\right]\left(G(z)-G_{\mathrm{sp}}(z)\right)=\frac{1}{24}$, corresponding to the fact that $K_{4}$ is the only cubic multigraph with 4 vertices which is not series-parallel. The limiting probability that $G\left(n, \frac{n}{2}\right)$ is series-parallel is

$$
p_{\mathrm{sp}}(0) \approx 0.98003
$$


See Figure 1 for a plot of $p_{\mathrm{sp}}(\lambda)$.

As another example, consider excluding $K_{3,3}$. Since the only 3-connected non-planar graph in $\operatorname{Ex}\left(K_{3,3}\right)$ is $K_{5}$, which is not cubic, the values of $h_{r}$ in this case are exactly the same as the ones in the planar case. Observe that 3-connectivity plays and important role in the equations of weighted cubic multigraphs in Section 3 (namely, the one related to the counting formula $H(z)$ ). Hence, the limiting probability of being in this class is exactly the same as of being planar, although $\operatorname{Ex}\left(K_{3,3}\right)$ is exponentially larger than the class of planar graphs (see Gerke et al. (2008)). But excluding the graph $K_{3,3}^{+}$, obtained by adding one edge to $K_{3,3}$, does increase the probability, since $K_{3,3}$ is in the class and is cubic and non-planar (the probability is computable since the 3-connected graphs in $\operatorname{Ex}\left(K_{3,3}^{+}\right)$are known, see Gerke et al. (2008)). Other classes such as $\operatorname{Ex}\left(K_{5}-e\right)$ or $\operatorname{Ex}\left(K_{3} \times K_{2}\right)$ can be analyzed too using the results from Giménez et al.

It would be interesting to compute the probability that $G(\lambda)$ has genus $g$. For this we need to count cubic multigraphs of genus $g$ (orientable or not). We only know how to do this for $g=0$, the reason being that a 3-connected planar graph has a unique embedding in the sphere. This is not at all true in positive genus. It is true though that almost all 3-connected graphs of genus $g$ have a unique embedding in the surface of genus $g$ (see Chapuy et al. (2011)). This could be the starting point for the enumeration, by counting first 3-connected maps of genus $g$ (a map is a graph equipped with a 2-cell embedding). But this is not enough here, since we need the exact numbers of graphs.

\section{Acknowledgements}

The first and third authors acknowledge the warm hospitality from the Laboratoire d'Informatique Algorithmique: Fondements et Applications (LIAFA) in Paris, where much of this work was done. The first and the third author also thank the support of the ANR project MAGNUM number 2010 BLAN-0204. The third author is also grateful to the ERC grant number 208471 - ExploreMaps.

\section{References}

M. Bodirsky, M. Kang, M. Löffler, and C. McDiarmid. Random cubic planar graphs. Random Structures Algorithms, 30(1-2):78-94, 2007.

B. Bollobás. The evolution of random graphs. Transactions of the American Mathematical Society, 286 (1):257-274, 1984a.

B. Bollobás. The evolution of sparse graphs. In Graph theory and combinatorics (Cambridge, 1983), pages 35-57. Academic Press, London, 1984b.

B. Bollobás. Random graphs. Academic Press Inc. [Harcourt Brace Jovanovich Publishers], London, 1985.

B. Bollobás, C. Borgs, J. T. Chayes, J. H. Kim, and D. B. Wilson. The scaling window of the 2-SAT transition. Random Structures Algorithms, 18(3):201-256, 2001.

G. Chapuy, É. Fusy, O. Giménez, B. Mohar, and M. Noy. Asymptotic enumeration and limit laws for graphs of fixed genus. Journal of Combinatorial Theory. Series A, 118(3):748-777, 2011. 
P. Erdős and A. Rényi. On the evolution of random graphs. Magyar Tud. Akad. Mat. Kutató Int. Közl., 5: 17-61, 1960.

P. Flajolet and R. Sedgewick. Analytic combinatorics. Cambridge University Press, Cambridge, 2009.

P. Flajolet, D. E. Knuth, and B. Pittel. The first cycles in an evolving graph. Discrete Mathematics, 75 (1-3):167-215, 1989. Graph theory and combinatorics (Cambridge, 1988).

P. Flajolet, B. Salvy, and G. Schaeffer. Airy phenomena and analytic combinatorics of connected graphs. Electronic Journal of Combinatorics, 11(1):Research Paper 34, 30 pp. (electronic), 2004.

A. Frieze and M. Krivelevich. On the non-planarity of a random subgraph. Preprint available on-line in arXiv:1205.6240.

S. Gerke, O. Giménez, M. Noy, and A. Weißl. The number of graphs not containing $K_{3,3}$ as a minor. Electronic Journal of Combinatorics, 15(1):Research Paper 114, 20, 2008.

O. Giménez, M. Noy, and J. Rué. Graph classes with given 3-connected components: asymptotic enumeration and random graphs. To appear at Random Structures and Algorithms.

S. Janson, D. E. Knuth, T. Łuczak, and B. Pittel. The birth of the giant component. Random Structures Algorithms, 4(3):231-358, 1993.

S. Janson, T. Łuczak, and A. Rucinski. Random graphs. Wiley-Interscience Series in Discrete Mathematics and Optimization. Wiley-Interscience, New York, 2000.

M. Kang and T. Łuczak. Two critical periods in the evolution of random planar graphs. Transactions of the American Mathematical Society, 364(8):4239-4265, 2012.

T. Łuczak, B. Pittel, and J. C. Wierman. The structure of a random graph at the point of the phase transition. Transactions of the American Mathematical Society, 341(2):721-748, 1994.

R. Otter. The number of trees. Annals of Mathematics (2), 49:583-599, 1948.

V. Stepanov. On some features of the structure of a random graph near a critical point. Theory of Probability \& Its Applications, 4(1):573-594, 1988.

E. M. Wright. The number of connected sparsely edged graphs. III. Asymptotic results. Journal of Graph Theory, 4(4):393-407, 1980. 
